\title{
Microsphere-assisted microscopy: from 2D to 3D super-resolution imaging
}

\author{
Paul Montgomery, Stéphane Perrin, Sylvain Lecler \\ Engineering science, computer science and imaging Laboratory (ICube), University of Strasbourg-CNRS, 23 rue \\ du Loess, 67037 Strasbourg, France \\ Tel: (+33) 388106231, e-mail: paul.montgomery@unistra.fr
}

\begin{abstract}
Here we present a new unlabelled far field optical imaging technique using microspheres to enhance the lateral resolution in optical microscopy. Both $2 \mathrm{D}$ and 3D super-resolution imaging using microspheres are considered, providing results of $2 \mathrm{D}$ and $3 \mathrm{D}$ imaging of surfaces with vastly improved lateral resolution and nanometric axial resolution for the 3D mode. Results are shown on narrow etched gratings in $\mathrm{Si}$, nanotextured stainless steel surfaces and Ag nanodots.

The main results demonstrate improvements of up to 3 times in lateral resolution, showing details of $300 \mathrm{~nm}$ for a microscope that would normally have a limit of $850 \mathrm{~nm}$. Microsphere-assisted microscopy is a major new type of far field optical microscopy with great potential for high resolution imaging, characterisation and nanometrology in materials science and the biological sciences.
\end{abstract}

Keywords: super-resolution, unlabelled microscopy, microspheres, gratings, simulations.

\section{INTRODUCTION}

High resolution optical microscopy is very attractive for use in materials and component characterization and the biological sciences because it is non-invasive, rapid and provides images over wide fields. One of the obvious drawbacks in optical microsocopy compared with SEM or AFM are the effects of diffraction that limit the lateral resolution to a value of about $200 \mathrm{~nm}$ in visible light [1].

Nonetheless, in recent decades, optical microscopy has been increasingly used for studying nanometric structures. In Ref. [2] we provided a survey of the different optical nanoscopy techniques that exist, pointing out that they can be divided into far field and near field imaging, and that far field imaging can be divided into two families: nanodetection, in which nanostructures can be studied without resolving all the details and superresolution, in which the features are resolved. This latter family can then be subdivided into labelled and unlabelled techniques, the latter being of great interest since they are non-invasive.

In 2011, the first reports were given of microsphere-assisted microscopy, using small glass microspheres (10 $\mu \mathrm{m}$ to $50 \mu \mathrm{m}$ in diameter) to considerably enhance the lateral resolution of an ordinary optical microscope [3]. The microsphere placed on the sample in front of the objective allows an increase in lateral resolution by a factor of between 3 to 5 times, claims of a lateral resolution of $50 \mathrm{~nm}$ being achieved in water immersion.

In the field of 3D surface metrology, it has been known for several decades that interference microscopy is capable of nanometric axial resolution, being used for characterizing microelectronic and optoelectronic devices as far back as the early 1990's [4]. Because of its ease of use, it has become a standard technique for surface roughness measurement [5]. Nonetheless, it has been frustrating that while the axial resolution is so high, the lateral resolution has remained limited by diffraction to values that are several orders of magnitude worse as in classical microscopy. Recently, several teams have succeeded in reducing this difference in scale by combining microsphere-assisted microscopy with interferometry to greatly improve the lateral resolution in surface roughness measurement [6-8]. In this work we present some of the latest results of our own work in this exciting new field of optical nanometrology. We show experimental results of high resolution measurements of narrow etched gratings, nanotextured structures on stainless steel using femtosecond lasers, and Ag nanodots on Si. Other results are also presented of simulations of microsphere imaging which help in the understanding of image formation.

\section{NOTIONS OF RESOLUTION}

The lateral resolution of an optical microscope is related to the optical parameters of the illumination, the optical performance of the imaging system and the means of detecting the light [1]. The lateral resolution can be calculated from the criterion $R_{c}=K \lambda / N A$, where $R_{c}$ is the distance between two point sources, $N A$ is the numerical aperture, with $N A=n \cdot \sin \theta$, and $\theta$ is the half angle of the light cone entering the objective. $n$ is the refractive index of the object-space and $K$ is $0.61,0.5$ or 0.47 depending on whether the resolution definition comes from the Rayleigh criterion, Abbe criterion or Sparrow criterion respectively. The criterion that is most commonly used is the Abbe criterion, leading to a typical value of $200 \mathrm{~nm}$ for the resolution limit for a wavelength of $400 \mathrm{~nm}$, a numerical aperture of 1.0 and an illumination which is angular and coherent (or incoherent). For 3D instruments, the concept of imaging resolution applies if we consider only features of shallow depth (several tens of $\mathrm{nm}$ ), in which case the classical imaging modulation transfer function (MTF) can 
be used. The experimental measurement of the 3D resolution of an optical profiler is therefore often performed with grating structures.

\section{Microsphere-assisted 2D microscopy}

Since Van Leeuwenhoek used millimetre-sized glass microspheres for early microscopy in 1675, the first work reported in more recent times of microsphere-assisted microscopy was in 2011 [3]. Small glass microspheres, 10 $\mu \mathrm{m}$ to $50 \mu \mathrm{m}$ in diameter are placed on the sample in front of the objective to considerably enhance the lateral resolution of an ordinary optical microscope (Figure 1). This optical arrangement allows an increase in lateral resolution by a factor of between 3 to 5 times, with claims of a lateral resolution of $50 \mathrm{~nm}$ being achieved.
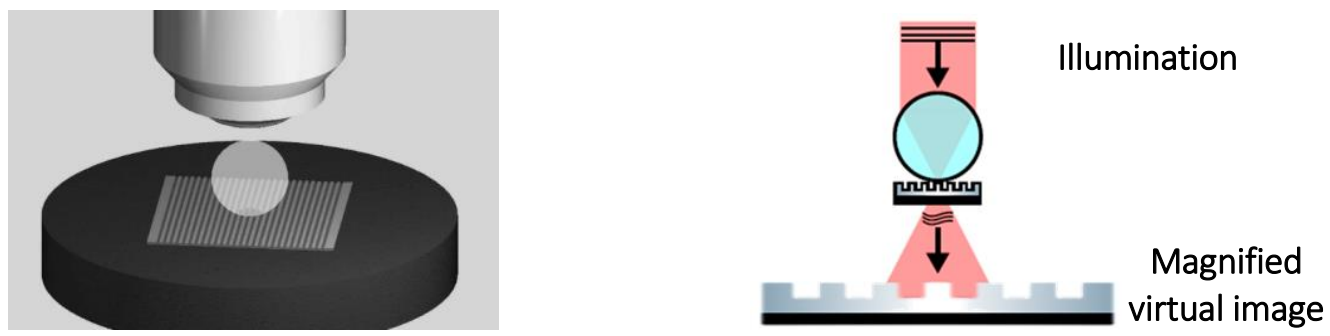

Figure 1. Optical arrangement (left) and magnification (right) in microsphere-assisted optical microscopy.

\subsection{Results of 2D imaging of microelectronic structures}

The results in Figure 2 show some examples of small silicon gratings and features on a microprocessor using a $\mathrm{x} 50$ objective $(\mathrm{NA}=0.55)$ in combination with a $24 \mu \mathrm{m}$ diameter microsphere placed on the sample in air. A grating period as small as $200 \mathrm{~nm}$, i.e. $\lambda / 3$, can be resolved through the microsphere, giving an equivalent numerical aperture of 1.5 in air, which is not visible directly by the objective. While the theoretical resolution of the objective alone has a value of $560 \mathrm{~nm}$, in practice this is closer to $850 \mathrm{~nm}$ in white light. The increase in resolution provided by the microsphere in this case is therefore a factor of 4.25 times.
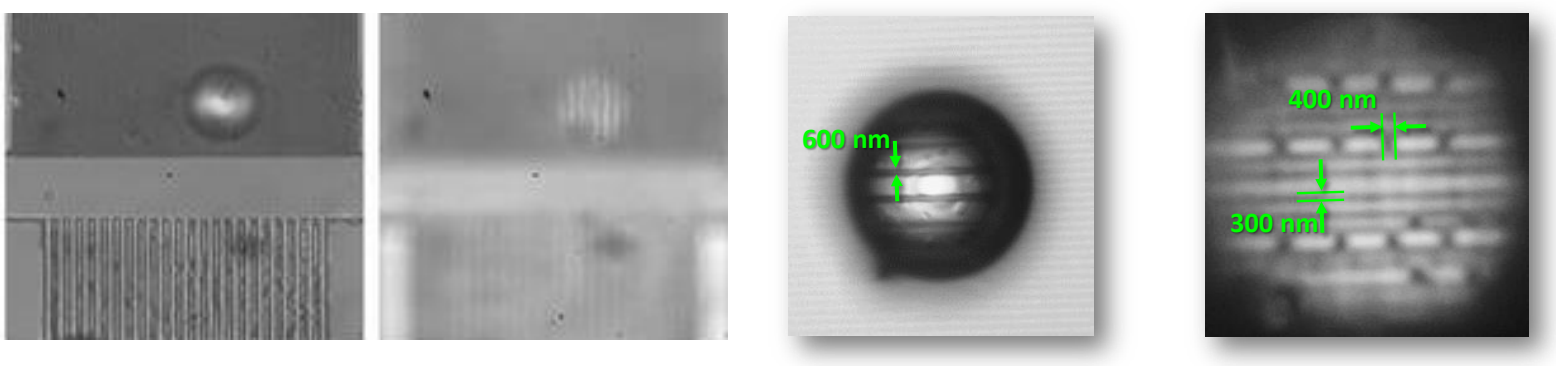

Figure 2. Improved resolution of gratings (left) and microelectronic structures (right) using microsphereassisted microscopy.

\subsection{D FEM simulations}

The optical phenomenon of microsphere imaging has been simulated in two dimensions in air using rigorous electromagnetic simulation with Comsol Multyphysics software [7]. An example is given in Figure 3 (left) of a $25 \mu \mathrm{m}$ diameter soda lime silica glass microsphere $(\mathrm{n}=1.53)$ with two spatially and temporally coherent point sources underneath it emitting at a wavelength of $400 \mathrm{~nm}$ and separated by $800 \mathrm{~nm}$.

The complex electric field above the microsphere is then time-reversed propagated in free space without the microsphere to retrieve the virtual image (Figure 3, right). The virtual images of the point sources correspond to the positions of the maximal intensities, the value along $\mathrm{Z}$ giving the position of the virtual image plane and the distance between the two maximal intensities along the $X$ axis giving the magnification. This has been performed for different diameters and wavelengths in order to investigate the imaging properties of the microsphere. 

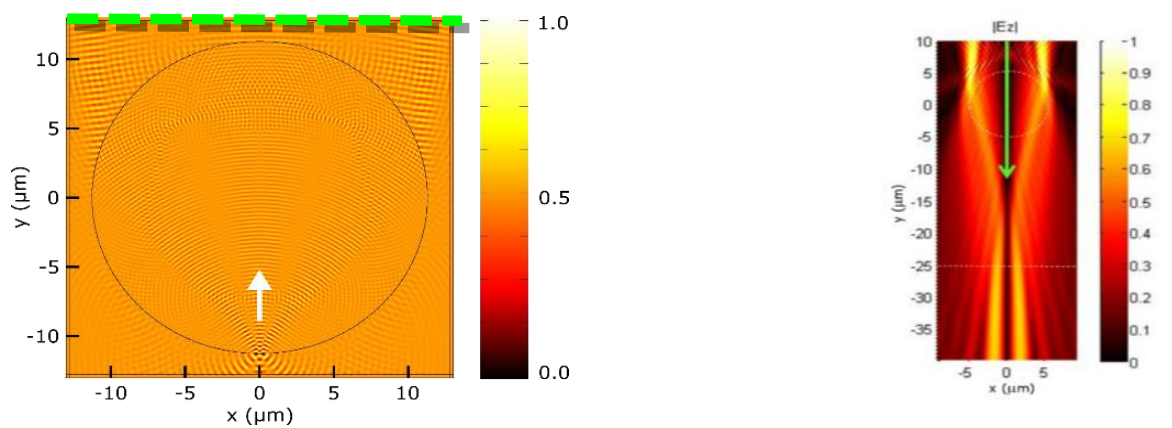

Figure 3. Numerical simulation of optical phenomenon in microsphere imaging: normalized real part of the electric fields from 2 point sources separated by $800 \mathrm{~nm}$ propagated through a 25 -mm diameter soda lime silica glass microsphere in air for $\lambda=400 \mathrm{~nm}$ (left) and normalized amplitude of the back-propagated electric field in free space for determining the resolution and magnification (right).

\section{MICROSPHERE-ASSISTED 3D MICROSCOPY}

Interference microscopy allows 3D surface roughness measurement with $\mathrm{nm}$ axial resolution but with a lateral resolution that is limited by the diffraction of light. By introducing a microsphere on the sample in front of the interference objective and adjusting the optical path lengths of the interferometer, a higher lateral resolution can be achieved for nanometrology. In the following we present results of measurements performed on different samples using a modified Leitz-Linnik interference microscope with white light illumination (centre wavelength of $560 \mathrm{~nm}$ ) from a white light LED, two x50 objectives in the Linnik configuration and a $24 \mu \mathrm{m}$ diameter glass microspheres placed on the sample. The enhanced imaging together with a classical five-step phase stepping algorithm for fringe analysis allows the reconstruction of the topography of the sub-diffraction-limit features [8].

\subsection{Measurements on gratings}

In order to test the performance of the microsphere imaging system, a set of calibrated standard gratings from SiMETRICS GmbH was first measured. This sample consists of a series of square profile gratings with pitches ranging from $6 \mu \mathrm{m}$ down to $300 \mathrm{~nm}$. An example of measurements of a $300 \mathrm{~nm}$ pitch grating is shown in Figure 4 that is not visible by the objective alone but is measurable through the microsphere.
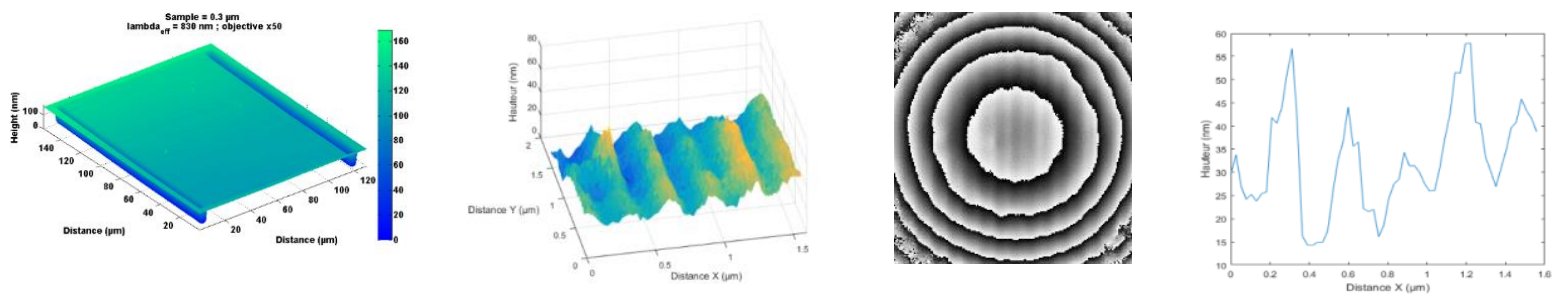

Figure 4. Measurements of a $0.3 \mu \mathrm{m}$ pitch RS-N grating with a $24 \mu \mathrm{m}$ microsphere and $x 50$ Leitz-Linnik $(N A=$ 0.3 ): measurement without and with microsphere, greyscale image and profile (left to right).

\subsection{Measurements on nanotextured stainless steel}

The next sample studied consists of self-organised ripples on stainless steel formed by illumination with femtosecond laser pulses in a one-step process (300 fs, $150 \mu \mathrm{J}, 200 \mathrm{kHz})$.
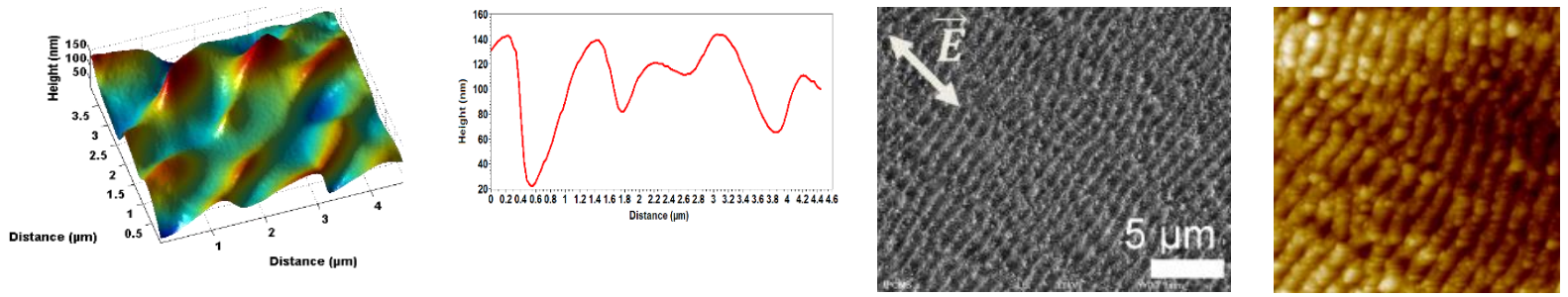

Figure 5. Microsphere measurements of nanoripples in stainless steel (left) and SEM and AFM (right).

These quasi-periodical structures have a spatial period of the order of the laser wavelength $(1030 \mathrm{~nm})$, and an orientation that is perpendicular to the direction of the incident linear polarization. Such nanostructured surfaces are used to change the visual aspect of the textured surface and applications in traceability and identification for 
anti-counterfeiting. The results shown in Figure 5 show some 3D measurements through a microsphere of some ripples and the equivalent images made with SEM and AFM for comparison.

\subsection{Measurements on Ag nanodots}

The final sample consists of an array of silver $(\mathrm{Ag})$ nano-dots covered by a silicon-oxynitride ( $\mathrm{SiON}$ ) layer on silicon made by the technique of nano imprint lithography. These nanostructures are used to obtain plasmonic resonance in order to increase the absorption in photovoltaic cells. AFM measurements (right) show that the nano-dots, are $200 \mathrm{~nm}$ wide, $300 \mathrm{~nm}$ long and $30 \mathrm{~nm}$ deep (Figure 6 right) and therefore too small to be resolved directly with the Linnik.
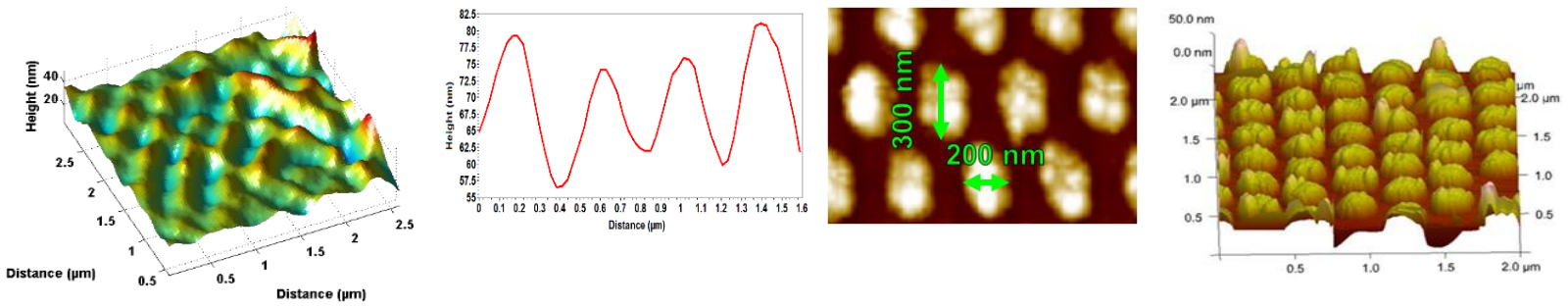

Figure 6. Microsphere measurements of silver nano-dots on silicon (left) and SEM and AFM (right).

Using a $24-\mu \mathrm{m}$ diameter glass microsphere in front of the $\mathrm{x} 50$ objective results in the ability to measure the nano-dots in 3D (Figure 6, left). Measuring the size of the measured nano-dots shows them to be $200 \mathrm{~nm}$ wide, $325 \mathrm{~nm}$ long and $20 \mathrm{~nm}$ to $25 \mathrm{~nm}$ deep. The lower heights found could be due to the super-resolution limit of the interference nanoscope (with the 24- $\mu$ m-diameter microsphere) which equals here the width of the nano-dots. Furthermore, the microscope objective must be able to resolve the details of the generated virtual image [9].

\section{CONCLUSIONS}

The techniques of 2D and 3D microsphere assisted microscopy have been presented. Microsphere imaging allows an increase in lateral resolution of optical microscopy by a factor of between $\mathrm{x} 3$ and $\mathrm{x} 5$ which means that resolutions of under $100 \mathrm{~nm}$ can be attained in air. Combined with interference microscopy, microsphere imaging shows a very high potential as a new nanometrology technique, conserving all the advantages of far field imaging in terms of being non-invasive and rapid. Results have been shown on gratings, microelectronic structures, nanoripples and silver nano-dots. Nonetheless, several challenges remain in order for the technique to become more widely used, including the need to better understand the imaging process, to reduce the measurement artefacts and to develop new ways of supporting and adjusting the microsphere in front of the microscope objective.

\section{ACKNOWLEDGEMENTS}

Thanks are extended to Camille Hairaye for providing the ripples in steel samples, to Thomas Fix for the silver nanodot samples and to the members of the $\mathrm{C}^{3}$-Fab platform for helping with the characterisation.

\section{REFERENCES}

[1] S. Perrin and P. Montgomery, Fourier optics: basic concepts, arXiv, Cornell University, 1802.07161, 2018.

[2] P. Montgomery and A. Leong-Hoi, Nanotechnology, Emerging optical nanoscopy techniques, Science and Applications 8, 31-44, 2015.

[3] Z. Wang, W. Guo, L. Li, B. Luk'yanchuk, A. Khan, Z. Liu, Z. Chen, and M. Hong, Optical virtual imaging at $50 \mathrm{~nm}$ lateral resolution with a white-light nanoscope, Nat. Commun., Vol. 2, p. 218, 2011.

[4] P.C. Montgomery, J.P. Fillard, M. Castagné and D. Montaner, Phase stepping microscopy (PSM): a qualification tool for electronic and optoelectronic devices, Semicond. Sci. Technol., Vol. 7, pp. A237A242, 1992.

[5] P. de Groot, Handbook of Optical Metrology: Principles and Applications, CRC Press, Boca Raton, 2015.

[6] F. Wang, L. Liu, P. Yu, Z. Liu, H. Yu, Y. Wang and W.J. Lib, Three-Dimensional Super-Resolution Morphology by Near-Field Assisted White-Light Interferometry, Nat. Sci. Rep. Vol. 6, pp. 24703, 2016.

[7] I. Kassamakov, S. Lecler, A. Nolvi, A. Leong-Hoi, P. Montgomery, E. Hæggström, 3D Super-Resolution Optical Profiling Using Microsphere Enhanced Mirau Interferometry, Nat. Sci. Rep., Vol. 7, p. 36832017.

[8] S. Perrin, A. Leong-Hoi, S. Lecler, P. Montgomery, Microsphere-assisted phase-shifting profilometry, Appl. Opt. Vol. 56, pp. 7249-7255, 2017.

[9] A. Darafsheh, N. I. Limberopoulos, J. S. Derov, D. E. Walker Jr, and V. N. Astratov, Advantages of microsphere-assisted super-resolution imaging technique over solid immersion lens and confocal microscopies, Applied Physics Letters, vol. 104, 061117, 2014. 